ANNALES

POLONICI MATHEMATICI

$98.1(2010)$

\title{
An intermediate value theorem in ordered Banach spaces
}

\author{
by Gerd Herzog (Karlsruhe)
}

\begin{abstract}
We prove an intermediate value theorem for certain quasimonotone increasing functions in ordered Banach spaces, under the assumption that each nonempty order bounded chain has a supremum.
\end{abstract}

1. Introduction. Let $E$ be a real Banach space ordered by a cone $K$. A cone $K$ is a nonempty closed convex subset of $E$ such that $\lambda K \subseteq K$ $(\lambda \geq 0)$, and $K \cap(-K)=\{0\}$. As usual $x \leq y: \Leftrightarrow y-x \in K$. For $x \leq y$ let $[x, y]$ denote the order interval of all $z$ with $x \leq z \leq y$. Let $K^{*}$ denote the dual wedge of $K$, that is, the set of all $\varphi \in E^{*}$ with $\varphi(x) \geq 0(x \geq 0)$.

For $D \subseteq E$ a function $f: D \rightarrow E$ is called quasimonotone increasing (in the sense of Volkmann [19]) if

$$
x, y \in D, x \leq y, \varphi \in K^{*}, \varphi(x)=\varphi(y) \Rightarrow \varphi(f(x)) \leq \varphi(f(y)) .
$$

For quasimonotone increasing functions several intermediate value (or equivalently fixed point) theorems are known, for special spaces [4], 8], 14, [15], under order conditions [6], 18, and under compactness conditions [6], [9], [10, [13, VIII.6], [18. For an application of such intermediate value theorems to boundary value problems see [7].

In this paper we will prove the following version under the assumption that the order defined by $K$ (or $K$ for short) has the following property:

(C) Each chain $C \subseteq E, C \neq \emptyset$, which is order bounded above has a supremum.

TheOREM 1. Let $E$ be ordered by a cone $K$ with property $(\mathrm{C})$, let $D \subseteq E$ be open, and let $f: D \rightarrow E$ be locally Lipschitz continuous and quasimonotone increasing. Moreover let $a, b \in D$ satisfy

$$
a \leq b, \quad[a, b] \subseteq D, \quad \text { and } \quad f(b) \leq 0 \leq f(a) .
$$

2010 Mathematics Subject Classification: 47H07, 47J20.

Key words and phrases: ordered Banach spaces, quasimonotone increasing functions, intermediate value property. 
Then

$$
\min f^{-1}(0) \cap[a, b] \quad \text { and } \max f^{-1}(0) \cap[a, b]
$$

exist.

Remarks. 1. Condition (C) is valid in particular if $K$ is regular (that is, each increasing and order bounded sequence in $E$ is convergent; see [2, Lemma 2] or [11, Lemma 1]). For regular cones a related intermediate value theorem is valid (see [6]). On the other hand, condition (C) implies that $K$ is normal (that is, $0 \leq x \leq y$ implies $\|x\| \leq \gamma\|y\|$ for some constant $\gamma \geq 1$; see [1, Lemma 2]), but normality for itself is not sufficient to guarantee the intermediate value property. We repeat the following example from [6] for the convenience of the reader:

Let $E=c(\mathbb{N}, \mathbb{R})$ be the Banach space of all convergent real sequences $x=\left(x_{k}\right)_{k \in \mathbb{N}}$, endowed with the supremum norm and ordered by the cone $K$ of all nonnegative sequences, which is normal. Let $f: E \rightarrow E$ be defined by

$$
f(x)=\left(0,1, x_{1}, x_{2}, x_{3}, \ldots\right)-x .
$$

Then $f$ is Lipschitz continuous and quasimonotone increasing, and

$$
f\left((1)_{k \in \mathbb{N}}\right)=(-1,0,0,0, \ldots) \leq 0 \leq(1,2,0,0,0, \ldots)=f\left((-1)_{k \in \mathbb{N}}\right),
$$

but $f(z)=0$ is unsolvable in $c(\mathbb{N}, \mathbb{R})$, since the only coordinatewise solution is

$$
z=(0,1,0,1,0,1, \ldots) .
$$

2. An example of a nonregular cone with property $(C)$ is the cone of all nonnegative sequences in $l^{\infty}(\mathbb{N}, \mathbb{R})$. More generally, let $J$ be a nonempty set and let $\left(F_{j}\right)_{j \in J}$ be a family of Banach spaces, each ordered by a regular cone $K_{j}$. Consider

$$
E=\left\{x=\left(x_{j}\right)_{j \in J}: x_{j} \in F_{j}(j \in J),\|x\|=\sup _{j \in J}\left\|x_{j}\right\|<\infty\right\}
$$

ordered by the cone

$$
K=\left\{x \in E: x_{j} \in K_{j}(j \in J)\right\} .
$$

Then $K$ has property (C) (see [11, Lemma 2]).

2. Preliminaries. To prove Theorem 1 we will make use of the following theorems. The first is a result on differential inequalities due to Volkmann [20, Satz 2], and two of its immediate consequences on dynamical systems:

TheOREM 2. Let $E$ be ordered by a cone $K$, let $D \subseteq E$ be open, let $f: D \rightarrow E$ be locally Lipschitz continuous and quasimonotone increasing, and let $u(\cdot, x):\left[0, T_{x}\right) \rightarrow D$ denote the solution of $u^{\prime}(t)=f(u(t)), u(0)=x$ (nonextendable to the right). Then: 
1. If $v, w:[0, T) \rightarrow D$ satisfy $v^{\prime}(t)-f(v(t)) \leq w^{\prime}(t)-f(w(t))(t \in[0, T))$ and $v(0) \leq w(0)$, then $v(t) \leq w(t)(t \in[0, T))$.

2. $x, y \in D, x \leq y \Rightarrow u(t, x) \leq u(t, y)\left(t \in\left[0, \min \left\{T_{x}, T_{y}\right\}\right)\right)$.

3. $x \in D, f(x) \geq 0[\leq 0] \Rightarrow t \mapsto u(t, x)$ is increasing [decreasing] on $\left[0, T_{x}\right)$.

Second, we will use the following versions of Bourbaki's and Tarski's fixed point theorems (see [3], [5, Proposition 1], [12]). For a function $g: \Omega \rightarrow \Omega$ we set

$$
\operatorname{Fix}(g):=\{x \in \Omega: g(x)=x\} .
$$

THEOREM 3. Let $\Omega \neq \emptyset$ be an ordered set such that each chain $\emptyset \neq$ $C \subseteq \Omega$ has a supremum. Let $g: \Omega \rightarrow \Omega$ satisfy $x \leq g(x)(x \in \Omega)$. Then $\operatorname{Fix}(g) \neq \emptyset$.

THEOREM 4. Let $\Omega \neq \emptyset$ be an ordered set such that $\min \Omega$ exists, and such that each chain $\emptyset \neq C \subseteq \Omega$ has a supremum. Let $g: \Omega \rightarrow \Omega$ be increasing. Then $\min \operatorname{Fix}(g)$ exists.

3. Proof of Theorem 1. We consider the set

$$
\Omega:=\left\{x \in[a, b]: f(x) \geq 0, x \leq z\left(z \in f^{-1}(0) \cap[a, b]\right)\right\} .
$$

First, observe that $a \in \Omega$, so $\Omega \neq \emptyset$. Next, let $x \in[a, b]$. According to Theorem 2 we have

$$
u(t, x) \in[a, b] \quad\left(t \in\left[0, T_{x}\right)\right) .
$$

If in addition $f(x) \geq 0$ then $t \mapsto u(t, x)$ is increasing on $\left[0, T_{x}\right)$, so

$$
f(u(t, x)) \geq 0 \quad\left(t \in\left[0, T_{x}\right)\right),
$$

and if in addition

$$
x \leq z\left(z \in f^{-1}(0) \cap[a, b]\right)
$$

then

$$
u(t, x) \leq u(t, z)=z \quad\left(t \in\left[0, T_{x}\right), z \in f^{-1}(0) \cap[a, b]\right) .
$$

Thus $x \in \Omega$ implies $u\left(\left[0, T_{x}\right), x\right) \subseteq \Omega$. Note that $u\left(\left[0, T_{x}\right), x\right)$ is a chain in $\Omega$ for each $x \in \Omega$.

Let $\emptyset \neq C \subseteq \Omega$ be a chain with $c:=\sup C$. We prove $c \in \Omega$. Clearly $c \in[a, b]$. According to Theorem 2 we have

$$
x \leq u(t, c) \quad\left(t \in\left[0, T_{c}\right), x \in C\right),
$$

and therefore

$$
c \leq u(t, c) \quad\left(t \in\left[0, T_{c}\right)\right) .
$$

Hence $u^{\prime}(0, c)=f(c) \geq 0$. Moreover

$$
x \leq z \quad\left(x \in C, z \in f^{-1}(0) \cap[a, b]\right),
$$

thus 


$$
c \leq z \quad\left(z \in f^{-1}(0) \cap[a, b]\right),
$$

and summing up we have $c \in \Omega$.

We define

$$
g: \Omega \rightarrow \Omega, \quad g(x)=\sup u\left(\left[0, T_{x}\right), x\right) .
$$

Now, $x \leq g(x)(x \in \Omega)$, and according to Theorem $3, g$ has a fixed point $\underline{z} \in \Omega$. Since $t \mapsto u(t, \underline{z})$ is increasing on $\left[0, T_{\underline{z}}\right)$ we conclude $T_{\underline{z}}=\infty$ and

$$
u(t, \underline{z})=\underline{z} \quad(t \in[0, \infty)),
$$

hence $f(\underline{z})=0$. To prove the minimality of $\underline{z}$ observe that $z \in[a, b], f(z)=0$ implies $\underline{z} \leq z$ by the definition of $\Omega$. Thus $\underline{z}=\min f^{-1}(0) \cap[a, b]$.

To prove the existence of a greatest solution in $[a, b]$ of $f(z)=0$ we consider

$$
h:-D \rightarrow E, \quad h(x)=-f(-x) .
$$

Now, $h$ is locally Lipschitz continuous, quasimonotone increasing, and

$$
h(-a) \leq 0 \leq h(-b) .
$$

Thus, in $[-b,-a]$ the equation $h(z)=0$ has a smallest solution $w$, and $\bar{z}:=-w=\max f^{-1}(0) \cap[a, b]$.

REMARK. If it is assumed in addition that $f(B)$ is bounded for each bounded subset $B \subseteq D$ then $T_{x}=\infty$ for each $x \in[a, b]$, and the proof above can be changed by applying Theorem 4 to $\Omega=\{x \in[a, b]: f(x) \geq 0\}$ and $g: \Omega \rightarrow \Omega$ defined by $g(x)=u(T, x)$ for any fixed $T>0$.

4. Discontinuous functions. Following the idea in [18] we can extend Theorem 1 the following way.

Let $D \subseteq E$ be open, let $a, b \in D$ satisfy $a \leq b,[a, b] \subseteq D$, and let

$$
F: D \times[a, b] \rightarrow E, \quad f:[a, b] \rightarrow E
$$

satisfy

(a) $x \mapsto F(x, y)$ is locally Lipschitz continuous and quasimonotone increasing for each $y \in[a, b]$,

(b) $y \mapsto F(x, y)$ is monotone increasing for each $x \in D$,

(c) $f(x)=F(x, x)(x \in[a, b])$, and $f(b) \leq 0 \leq f(a)$.

Under these assumptions $f$ is quasimonotone increasing, and allows upward jumps (see [18]). We have

TheOREM 5. Let $E$ be ordered by a cone $K$ with property $(\mathrm{C})$, let $D \subseteq E$ be open, let $a \leq b$ with $[a, b] \subseteq D$, and let $F: D \times[a, b] \rightarrow E$ and $f:[a, b] \rightarrow$ E satisfy (a)-(c) above. Then

$$
\min f^{-1}(0) \cap[a, b] \quad \text { and } \quad \max f^{-1}(0) \cap[a, b]
$$

exist. 
5. Proof of Theorem 5. Let $y \in[a, b]$. Then

$$
F(b, y) \leq f(b) \leq 0 \leq f(a) \leq F(a, y) .
$$

According to Theorem 1 the mapping $x \mapsto F(x, y)$ has in $[a, b]$ a smallest zero $g(y)$. We obtain a function $g:[a, b] \rightarrow[a, b]$ and we prove that $g$ is increasing. Indeed, let $y, z \in[a, b]$ with $y \leq z$. Now

$$
F(g(z), y) \leq F(g(z), z)=0 \leq F(a, y) .
$$

Thus $x \mapsto F(x, y)$ has in $[a, g(z)]$ a zero $v$, which is a zero in $[a, b]$. Therefore

$$
g(y) \leq v \leq g(z)
$$

According to Theorem 4 (applied to $\Omega=[a, b]) \underline{z}:=\min \operatorname{Fix}(g)$ exists, and clearly $f(\underline{z})=0$. Now, let $z \in[a, b]$ satisfy $f(z)=0$. Then $z$ is a zero of $x \mapsto F(x, z)$ in $[a, b]$, hence $g(z) \leq z$. Thus $g([a, z]) \subseteq[a, z]$, and so $g$ has a fixed point $w$ in $[a, z]$ which is a fixed point in $[a, b]$. Thus

$$
\underline{z}=\min \operatorname{Fix}(g) \leq w \leq z .
$$

Therefore $\underline{z}=\min f^{-1}(0) \cap[a, b]$.

Application of this state of knowledge to $H:(-D) \times[-b,-a] \rightarrow E$ and $h:[-b,-a] \rightarrow E$ defined by

$$
H(x, y)=-F(-x,-y), \quad h(x)=H(x, x)
$$

proves the existence of $\bar{z}=\max f^{-1}(0) \cap[a, b]$.

6. Example. Let $F$ be a Banach space ordered by a regular cone $K_{F}$ with nonempty interior, let $E=l^{\infty}(\mathbb{Z}, F)$ be ordered by the cone

$$
K=\left\{\left(x_{n}\right)_{n \in \mathbb{Z}}: x_{n} \in K_{F}(n \in \mathbb{Z})\right\},
$$

and let $q: F \rightarrow F$ be locally Lipschitz continuous and quasimonotone increasing. We can apply Theorem 1 to prove

Theorem 6. Let $\left(w_{n}\right)_{n \in \mathbb{Z}} \in E$, and let $a, b \in F$ be such that

$$
a \leq b, \quad q(b) \leq w_{n} \leq q(a) \quad(n \in \mathbb{Z}) .
$$

Then the second order difference equation

$$
z_{n+1}-2 z_{n}+z_{n-1}+q\left(z_{n}\right)=w_{n} \quad(n \in \mathbb{Z})
$$

has in $\left[(a)_{n \in \mathbb{Z}},(b)_{n \in \mathbb{Z}}\right]$ a smallest and a greatest solution.

Proof. According to Remark 2. the order on $E$ defined by $K$ has property (C). We consider $f: E \rightarrow E$ defined by

$$
f\left(\left(x_{n}\right)_{n \in \mathbb{Z}}\right)=\left(x_{n+1}-2 x_{n}+x_{n-1}+q\left(x_{n}\right)-w_{n}\right)_{n \in \mathbb{Z}} .
$$

It is clear that $f$ is locally Lipschitz continuous and, using Uhl's criterion for quasimonotonicity [17, Theorem 2], it is not hard to see that $f$ is quasi- 
monotone increasing. We have

$$
f\left((b)_{n \in \mathbb{Z}}\right)=\left(q(b)-w_{n}\right)_{n \in \mathbb{Z}} \leq(0)_{n \in \mathbb{Z}} \leq\left(q(a)-w_{n}\right)_{n \in \mathbb{Z}}=f\left((a)_{n \in \mathbb{Z}}\right) .
$$

Thus, according to Theorem 1 , the maximum and the minimum of

$$
f^{-1}\left((0)_{n \in \mathbb{Z}}\right) \cap\left[(a)_{n \in \mathbb{Z}},(b)_{n \in \mathbb{Z}}\right]
$$

exist.

Consider for example $F=\mathbb{R}^{3}$ ordered by the ice-cream cone

$$
K_{F}=\left\{x=(\xi, \eta, \zeta): \zeta \geq \sqrt{\xi^{2}+\eta^{2}}\right\}
$$

and $q: F \rightarrow F$ defined by

$$
q(\xi, \eta, \zeta)=\left(\begin{array}{c}
-\eta-2 \xi \zeta \\
\xi-2 \eta \zeta \\
-\xi^{2}-\eta^{2}-\zeta^{2}
\end{array}\right)
$$

Obviously $q$ is locally Lipschitz continuous, and $q$ is quasimonotone increasing since $q^{\prime}(\xi, \eta, \zeta): \mathbb{R}^{3} \rightarrow \mathbb{R}^{3}$ is a linear quasimonotone increasing mapping for each $(\xi, \eta, \zeta) \in \mathbb{R}^{3}$ (see [16, Theorem 3.31]). Since $p=(0,0, \lambda) \in \operatorname{Int} K$ for each $\lambda>0$, and since $q(0,0, \lambda)=-\left(0,0, \lambda^{2}\right)$ we can apply Theorem 6 if $\left(w_{n}\right)_{n \in \mathbb{Z}}$ is a bounded sequence in $-K_{F}$, by setting

$$
a=(0,0,0), \quad b=(0,0, \lambda),
$$

with $\lambda>0$ sufficiently large.

\section{References}

[1] T. Andô, On fundamental properties of a Banach space with a cone, Pacific J. Math. 12 (1962), 1163-1169.

[2] V. A. Bondarenko, Integral inequalities for a Volterra equation in a Banach space with a cone, Mat. Zametki 9 (1971), 151-160 (in Russian).

[3] N. Bourbaki, Sur le théorème de Zorn, Arch. Math. (Basel) 2 (1951), 434-437.

[4] B. Choudhary and S. K. Mishra, A note on fixed points for discontinuous quasimonotone maps in sequence spaces, Indian J. Math. 37 (1995), 263-268.

[5] M. Erné, $\mathcal{W}$-completeness and fixpoint properties, Arch. Math. (Brno) 24 (1988), $147-155$.

[6] G. Herzog and R. Lemmert, Intermediate value theorems for quasimonotone increasing mappings, Numer. Funct. Anal. Optim. 20 (1999), 901-908.

[7] - - - Boundary value problems via an intermediate value theorem, Glasgow Math. J. 50 (2008), 531-537.

[8] S. Hu, Fixed points for discontinuous quasi-monotone maps in $\mathbb{R}^{n}$, Proc. Amer. Math. Soc. 104 (1988), 1111-1114.

[9] V. Lakshmikantham, Monotone flows and fixed points for dynamic systems on time scales in a Banach space, Appl. Anal. 56 (1995), 175-184.

[10] V. Lakshmikantham and B. Kaymakalan, Monotone flows and fixed points for dynamic systems on time scales, in: Advances in Difference Equations, Comput. Math. Appl. 28 (1994), 185-189. 
[11] R. Lemmert, R. M. Redheffer and P. Volkmann, Ein Existenzsatz für gewöhnliche Differentialgleichungen in geordneten Banachräumen, in: General Inequalities, 5 (Oberwolfach, 1986), Int. Schriftenreihe Numer. Math. 80, Birkhäuser, Basel, 1987, 381-390.

[12] G. Markowsky, Chain-complete posets and directed sets with applications, Algebra Universalis 6 (1976), 53-68.

[13] R. H. Martin, Nonlinear Operators and Differential Equations in Banach Spaces, Krieger, Malabar, 1987.

[14] I. Redheffer und P. Volkmann, Ein Fixpunktsatz für quasimonoton wachsende Funktionen, Arch. Math. (Basel) 70 (1998), 307-312.

[15] S. Schmidt, Fixed points for discontinuous quasimonotone maps in sequence spaces, Proc. Amer. Math. Soc. 115 (1992), 361-363.

[16] R. J. Stern and H. Wolkowicz, Exponential nonnegativity on the ice cream cone, SIAM J. Matrix Anal. Appl. 12 (1991), 160-165.

[17] R. Uhl, Ordinary differential inequalities and quasimonotonicity in ordered topological vector spaces, Proc. Amer. Math. Soc. 126 (1998), 1999-2003.

[18] —, Smallest and greatest fixed points of quasimonotone increasing mappings, Math. Nachr. 248-249 (2003), 204-210.

[19] P. Volkmann, Gewöhnliche Differentialungleichungen mit quasimonoton wachsenden Funktionen in topologischen Vektorräumen, Math. Z. 127 (1972), 157-164.

[20] —, Über die Invarianz konvexer Mengen und Differentialungleichungen in einem normierten Raume, Math. Ann. 203 (1973), 201-210.

Gerd Herzog

Institut für Analysis

Universität Karlsruhe

D-76128 Karlsruhe, Germany

E-mail: Gerd.Herzog@math.uni-karlsruhe.de

Received 7.4.2009

and in final form 21.9.2009 
